\title{
Control de calidad de plantas medicinales y su legislación sanitaria en México
}

\section{Quality control of medicinal plants and its health legislation in Mexico}

\author{
Araceli Pérez-Vásquez ${ }^{1 \star}$ Erika Vivani Castillejos-Ramírez ${ }^{2}$ \\ y Jakziri Guadalupe Pérez-Herrera ${ }^{3}$
}

Fecha de recepción: 5 de diciembre de 2018

Fecha de aceptación: 6 de marzo de 2019

\begin{abstract}
Resumen - México es un país con una larga tradición en el consumo de plantas medicinales, sin embargo, aún falta mucho por hacer en cuestiones de su regulación y protección. Y es que una gran cantidad de plantas usadas en las prácticas médicas tradicionales son objeto de un activo comercio nacional e internacional, constituyéndose así un mercado global altamente demandante. En este contexto, las plantas medicinales que forman parte de los productos herbolarios (como drogas crudas) o que son base principal de los mismos (siendo sus drogas crudas el principal componente) deben someterse a un examen riguroso mediante diversas técnicas analíticas, de acuerdo con la reglamentación sanitaria vigente, esto con la finalidad de establecer su identidad, composición y pureza. Estas determinaciones son la base de los ensayos de calidad. Desde el punto de vista de la sustentabilidad, el control de calidad está encaminado a contribuir a la conservación, manejo y uso de especies vegetales en peligro de extinción o especies amenazadas que tienen una alta demanda comercial en la población.
\end{abstract}

\section{Palabras clave:}

Plantas medicinales, control de calidad, productos herbolarios.
Abstract - Mexico is a country with a long tradition in the consumption of medicinal plants. However, there is still a lot to do regarding its regulation and protection, since a huge number of plants used in traditional Mexican medicines are subject to an active national and international trade, thus constituting a highly demanding global market. In this context, the medicinal plants that constitute one part of herbal products -as raw materials- or the main part -with its raw material-, must undergo a rigorous assessing by using analytical techniques in compliance with the sanitary regulations in force, to establish their identity, purity and content. All these test procedures are the basis of quality control for herbal drugs. From a sustainability perspective, quality control is aimed to contribute to the conservation, management and use of endangered plant species or threatened species that have high commercial demand in the population.

\section{Keywords:}

Medicinal plants, quality control, herbal products.

\footnotetext{
${ }^{1}$ Doctora en Ciencias (Químicas) con especialidad en Fitoquímica y Farmacognosia por la UNAM. Profesora de Tiempo completo en la Facultad de Química de la UNAM y profesora de asignatura, Facultad de Ciencias Químicas, UABJO. Miembro del SNI nivel 1. *correo electrónico: perezva@unam.mx 2 Maestra en Ciencias (Químicas) por la UNAM. Candidata a Doctora en Ciencias Químicas, con especialidad en Fitoquímica y Farmacognosia por el Posgrado en Ciencias Químicas de la UNAM. Correo electrónico: erikavivani@gmail.com

${ }^{3}$ Pasante de Químico Farmacéutico Biólogo. Facultad de Ciencias Químicas, UABJO. Correo electrónico: guajak@hotmail.com
} 


\section{Importancia del control de la calidad de plantas medicinales}

E El uso de plantas medicinales ha crecido sustancialmente en los últimos años. Se estima que $80 \%$ de las personas en el mundo recurren a ellas para procurar la salud, aliviar algunos síntomas o curarse (Rajani y Kanaki, 2008). Para mucha gente las plantas representan un recurso inmediato y acuden a mercados, tianguis, droguerías, expendios de plantas medicinales para conseguirlas o alguna persona se las proporciona. También, el consumo de suplementos alimenticios elaborados con plantas o parte de ellas, así como de remedios que se presentan como medicamentos, ha crecido enormemente. Muchos de estos productos se promocionan con las características de curar diferentes padecimientos; sin embargo, tienen algo en común: la mayoría no incluye la información científica necesaria que garantice su calidad, es decir, que se tomen en cuenta factores extrínsecos e intrínsecos para su producción, como son -en el caso de los extrínsecos- una correcta identificación y autentificación de la planta, impurezas que pueda contener (plaguicidas, micotoxinas y demás sustancias tóxicas) asociadas a toda la cadena de producción; así como factores intrínsecos, entre ellos el tipo de metabolitos y la proporción de éstos, los cuales contribuyen en la actividad terapéutica o en la toxicidad. Las anteriores consideraciones son fundamentales para establecer la calidad de las plantas medicinales 0 en productos elaborados a base de ellas, y esta calidad incide directamente en su eficacia y seguridad (inocuidad), razón por la que representan un enorme desafío para las autoridades de salud que tienen que regularlas. En el caso de México, la Comisión Federal para la Protección contra Riesgos Sanitarios (Cofepris), órgano desconcentrado de la Secretaría de Salud, ha enfrentado y abordado este problema al establecer la regulación, el control y el fomento sanitario en lo relativo a dichos productos (Gómez Castellanos, 2009; Ortega, Phail y VegaMontiel, 2011).
Por otra parte, el uso de plantas medicinales ya no sólo está destinado a procurar la salud, sino que también se utilizan para "reforzar defensas", "porque son naturales", "porque no le hacen daño a mi organismo y me dan muchos nutrientes". No obstante, y sacando a las plantas de su contexto etnocultural en el cual se encuentran inmersas en las comunidades rurales e indígenas de México y en donde -como lo menciona Antonio Barrera (1992, p. 186)- "[...] persiste su uso, dado que forman parte de un sistema médico más accesible que se mantiene porque es económico, por la estrecha relación entre el origen de la enfermedad y la religión cristiana y por coherencia cultural", el consumo se ha extendido enormemente y de manera irracional en todas las ciudades, estados y países, constituyéndose un mercado activo y con un crecimiento económico del 15\% anual, en promedio (Srirama et al., 2017).

En este contexto, y en un enorme esfuerzo por asegurar el uso de las plantas medicinales para el bien de las comunidades, la Organización Mundial de la Salud (OMS) ha instado a sus países miembro, entre los que se encuentra México, a que certifiquen la calidad de las especies vegetales que se comercializan (OMS, 2011). Al respecto, definió su papel en el uso, manejo y cultivo de plantas medicinales incluyéndolas en un documento que tituló "Estrategia de la OMS sobre medicina tradicional 2002-2014", el cual abordó temas relacionados con la política, seguridad, eficacia, calidad, acceso y uso racional de los recursos naturales destinados a emplearse en la medicina tradicional, complementaria y alternativa (OMS, 2002). Actualmente se encuentra vigente una segunda actualización de esta estrategia, la cual tiene competencia hasta el 2023 (OMS, 2013) y en la que se insta a los estados miembros a que se comprometan y asuman la responsabilidad crucial de proteger la salud de sus habitantes, para lo cual "deberán velar por la seguridad de las prácticas de la medicina tradicional y prevenir más eficazmente los riesgos identificados de esas prácticas" (OMS, 2013, p. 31). Los riesgos a los que se refiere este documento están relacionados, entre otros, con el mal uso que se puede dar a las plantas 
medicinales, como son las adulteraciones, falsificaciones o productos de mala calidad que podrían causar graves efectos a la salud (Posadzki, Watson y Ernst, 2013; Ahmed y Hasan, 2015).

\section{El caso de las adulteraciones o falsificaciones}

Una adulteración o falsificación de plantas medicinales con gran valor comercial puede ocurrir por varios motivos. Así, por ejemplo, la especie vegetal Matricaria recutita, conocida comúnmente como manzanilla, es recomendada para diferentes tipos de padecimientos que no están directamente relacionados, sin embargo, y debido a su alto consumo es una especie vegetal que se sustituye por otras, sufriendo de esta manera adulteraciones o falsificaciones a nivel mundial (Das, 2014). En 1919, Clevenger y Ewing documentaron la primera falsificación de dicha especie con otra parecida llamada Santolina chamaecyparissus, cuyo nombre común es "manzanilla fina" (Clevenger y Ewing, 1919). Como los autores lo mencionan, el suministro de manzanilla disminuyó considerablemente en la primera guerra mundial. Alemania era uno de los principales países que suministraba grandes cantidades de esta planta al resto de Europa, sin embargo y como las fronteras se cerraron, la distribución de esta planta se vio enormemente afectada y esto propició el aumento de las adulteraciones y falsificaciones (Clevenger y Ewing, 1919). Hasta el día de hoy se han detectado otras especies, sobre todo de los géneros Chamaemelum, Bellis, Chrysanthemum y Tanacetum (Guzelmeric, Vovk y Yesilad, 2015), como adulterantes o falsificantes de manzanilla; todas ellas tienen en común que pertenecen a la misma familia botánica (Asteraceae) y pueden presentar inflorescencias blancas.

En cuanto al uso medicinal de una especie vegetal, de éstas se pueden ocupar todas sus partes o alguna de ellas; es posible utilizar las hojas, tallos, raíces cortezas, flores o semillas, entre otras. Sin embargo, a pesar de que los tallos, hojas, flores y raíces provengan de la misma planta, la única parte que debe ser empleada es la que se reconoce con el uso medicinal y la que recibe el nombre de droga, y si se presenta en forma seca se denomina droga cruda (Evans, 2009). Un caso importante de adulteración o falsificación relacionado con la droga cruda es el de las cortezas. En México, quizás una de las plantas más comercializadas y recomendadas debido a sus múltiples bondades es el tepezcohuite y su droga cruda es la corteza seca, presentada en trozos de la especie medicinal Mimosa tenuiflora (Martínez y López Herrera, 1995; Rivera-Arce et al., 2007). Debido a la gran fama que ha adquirido por sus propiedades medicinales tan importantes para curar heridas, infecciones de la piel y quemaduras (Martínez y López Herrera, 1995; CadenaIñiguez, Cruz-Morales y Ballinas-Albores, 2014), es una de las cortezas más adulteradas con otras especies que presentan similitudes morfológicas. Las especies que se han reportados adulterantes de $M$. tenuiflora son Mimosa arenosa, Acacia pennatula, Byrsonima crassifolia, Luehea candida y Guazuma ulmifolia (Rivera-Arce et al., 2007). Una caso similar ocurre con Amphipterygium adstringens, especie endémica de México cuya droga cruda, que también es una corteza, es conocida popularmente como cuachalalate. Es una de las plantas medicinales más comercializadas en el centro y sur del país, situación que conduce a su adulteración con especies como Cyrtocarpa procera, gracias a la semejanza que existe entre las cortezas de ambas especies (Rosas-Acevedo et al., 2011).

Por otra parte, las adulteraciones o falsificaciones también se pueden dar por la gran confusión que existe en el uso de nombres vernáculos de las plantas medicinales usados entre diferentes grupos indígenas, regiones, países o continentes. Un caso es el que se presenta en México y otros países como Brasil y Estados Unidos, donde la especie Passiflora incarnata es utilizada como sedante y ansiolítica, pero el elevado número de especies del género Passiflora (P. actinia, $P$. alata, $P$. amethystina, $P$. capsularis y $P$. cincinnata, entre otras) y el nombre común (flor de la pasión) que se le 
asigna a la mayoría de ellas conducen a la adulteración y falsificación de $P$. incarnata con otras especies (Wosch et al., 2015). Otro ejemplo sería el nombre que designa a árnica, que en el caso de la árnica mexicana esta hace referencia a Heteroteca inuloides y la droga cruda en nuestro país puede ser tanto las flores secas (que es considerada en la Farmacopea Herbolaria de los Estados Unidos Mexicanos) como los tallos y hojas en mezclas, que en su conjunto se denominan partes aéreas (Delgado et al., 2001). También en México es conocida como árnica la especie Tithonia diversifolia, aunque a veces se le denomina a esta especie "girasol mexicano o hierba de la caléndula" (Owoyele, Wuraola, Soladoye y Olaley, 2004). Por otra parte, en Europa se conoce como árnica o "verdadera árnica" a la especie Arnica montana (Waizel-Bucay y CruzJuárez, 2014); en este continente, la árnica mexicana es conocida como "falsa árnica", de tal manera que esta especie tan importante en México, se presenta como adulterante o en muchos casos falsificante de la "verdadera árnica" (Gafner y Applequist, 2016). A este respecto, es necesario definir a qué nos referimos con una adulteración o falsificación en cuestión de plantas medicinales. De acuerdo con Foster (2011), la adulteración tiene que ver con la detección en la droga cruda de restos de otras plantas o de la adición de alguna otra sustancia que simule color, olor o sabor en los productos herbolarios. También, la adulteración puede ser debida a la adición de fármacos, en especial aquellos productos empacados y comercializados como "a base de hierbas" que se expenden mundialmente, promocionándose a través de los medios de comunicación social que tienen como destinatario al público en general (Rocha, Amaral y Oliveira, 2016). El ejemplo más claro y más grave debido a su alto consumo son aquellos que dicen tener un efecto adelgazante. En estos "productos a base de hierbas" se han encontrado fármacos como el fenproporex, el cual está clasificado como adrenérgico de tipo anfetamina (Mariotti, Rossato, Fröehlich y Limberger, 2013). Este fármaco se emplea en el tratamiento de la obesidad por su efecto anorexígeno, ya que funciona como supresor del apetito (Calahan et al., 2016; Mariotti, Rossato, Fröehlich y Limberger, 2013). Por otra parte, la falsificación se refiere a la sustitución total de la droga cruda obtenida de la especie vegetal y generalmente se ofrece o etiqueta premeditadamente como algo que no es (Foster, 2011); un ejemplo de falsificación de especies ocurre con la cúrcuma (Curcuma longa), que es altamente comercializada a nivel mundial en diferentes presentaciones, una de ellas es en forma de polvo, situación que la vuelve susceptible a falsificaciones con materiales de relleno que pasan desapercibidos visualmente. Dentro de los falsificantes más comunes se encuentran el polvo de la Curcuma cedoaria y almidón proveniente de Manihot esculenta (Parvathy, Swetha, Sheeja y Sasikumar, 2015). En algunos casos, la poca disposición de las drogas crudas o la escasez de las mismas en regiones altamente demandantes originan que se adulteren. Es el caso de la hierba de San Juan (Hypericum perforatum) que es cultivada y comercializada en Europa; no obstante, en países como la India, la carencia de esta especie y la abundancia de Hypericum patulum ha provocado que esta última se venda cotidianamente como $\mathrm{H}$. perfotarum (Mitra y Kannan, 2007). Las adulteraciones y falsificaciones son, por lo tanto -como lo menciona Selvam (2010)-, una actividad fraudulenta o mala praxis relacionada con un engaño premeditado para lucrar con productos a base de hierbas que no tienen lo que dicen que contienen y que por ello pueden poner en riesgo a los consumidores (Selvam, 2010).

Es importante mencionar que, de acuerdo con la reglamentación vigente, los productos herbolarios sólo deberán hacer referencia a las drogas vegetales reconocidas como tal. La OMS, en su publicación sobre normas de calidad para materiales de plantas medicinales, recomienda rechazar cualquier lote de materia prima que contenga otra parte de la misma planta (por ejemplo, tallos en vez de hojas), aun cuando hayan derivado de la auténtica (OMS, 2011). 
Al respecto, los ensayos para establecer la calidad de las especies se vuelven de carácter obligatorio para asegurar su autenticidad y además, determinar adulterantes o falsificantes de drogas crudas.

\section{Ensayos de calidad}

La calidad, de acuerdo con la norma NOM-001-SSA-2010, se define como el "cumplimiento de especificaciones establecidas para garantizar la aptitud de uso". De acuerdo a esta definición y tomando a las plantas medicinales como materia prima para preparar productos herbolarios, la calidad consiste en la ejecución de las determinaciones necesarias para conocer su identidad, composición y pureza, y son establecidas para garantizar la eficacia y la seguridad, lo cual implica que tengan que someterse a una serie de ensayos tanto las drogas crudas como los extractos obtenidos de las plantas en estudio. La calidad debe ser prioridad en todas las etapas de obtención del producto herbolario como son, el cultivo, la cosecha, el empaque, el etiquetado y la presentación final (Yau, Goh y Koh, 2015). Los ensayos de calidad se encuentran descritos en documentos establecidos para tal fin como son: farmacopeas, normas nacionales e internacionales, monografías de la OMS y monografías científicas. En el caso de México, somos uno de los pocos países en el mundo en contar con una Farmacopea Herbolaria (Secretaría de Salud, 2013), donde se compilan las determinaciones analíticas requeridas para demostrar la calidad de la especie vegetal en estudio. Estas determinaciones son las siguientes:

1. Ensayos destinados a establecer la identidad botánica y química.

2. Ensayos enfocados a establecer su composición a partir de la valoración del contenido de marcadores y/o principios activos.

3. Ensayos destinados a conocer su pureza, como es la especificación de material extraño, contenido de plaguicidas, tipo de aflatoxinas y de metales pesados.

Los primeros ensayos que se realizan son los conducentes a establecer la identidad botánica. Estos están destinados a establecer la autenticidad de la planta partiendo del reconocimiento de sus características macroscópicas como es la parte de la planta, así como de sus características microscópicas (histológicas) y en el caso que se requiera, características organolépticas, todo respecto de la información taxonómica que se tenga de la especie (Khan y Smillie, 2012). Asimismo, deben documentarse cada uno de los nombres comunes, lo relacionado a variedades (taxonómicas), quimiotipos y formas taxonómicas, si existen (OMS, 2011). Los ensayos de identidad química son prioritarios para determinar cualitativamente el grado de adulteración o la falsificación que se está haciendo de una droga cruda. Dado que los ensayos de identidad química, así como los de valoración hacen uso del contenido metabólico de las plantas, es importante primeramente realizar los estudios fitoquímicos necesarios en las plantas en estudio. Es conveniente mencionar que las plantas biosintetizan una gran cantidad de compuestos estructuralmente diversos y aquellos que no participan directamente en su desarrollo y crecimiento se denominan metabolitos secundarios; éstos también reciben el nombre de productos naturales (Williams, Stone, Hauck y Rahman, 1989), los cuales, basados en su origen biosintético, pueden ser terpenoides, alcaloides o fenilpropanoides, entre otros (Croteau, Kutchan y Lewis, 2000). Así, los productos naturales pueden presentar estructuras de tipo lactonas sesquiterpénicas, esteroides, taninos 0 flavonoides, por mencionar los más comunes. Estos metabolitos de las plantas son los que principalmente les confieren la actividad farmacológica relacionada con su uso etnomédico (Salim, Chin, y Kinghorn, 2008). También, estos metabolitos son elegidos para desarrollar e implementar los métodos analíticos útiles para los ensayos farmacopéicos de identidad química y composición, y en este caso reciben el nombre de marcadores químicos (Li et al., 2008). Así, el término de marcadores hace referencia a un compuesto o grupo de compuestos que puede estar presente en la planta de manera natural (metabolito) y puede ser el que le otorgue la actividad biológica, el cual se denomina 
marcador activo, o también el que se encuentra en buena cantidad y/o es de restricción taxonómica, que se puede denominar marcador analítico. También es posible que sea tóxico para el humano, pero que resulte fundamental para la planta, a lo cual se le denomina marcador negativo (Li et al., 2008). En todos los casos, las matrices que se examinan son los extractos obtenidos de las plantas y los analitos serán los marcadores que se seleccionen. Los ensayos destinados a establecer la composición están encaminados a "valorar el contenido", es decir, a conocer la cantidad de los metabolitos o marcadores de las especies vegetales en relación con una proporción (porcentaje). Su contenido representa, en muchas ocasiones, la cantidad a la que tienen que estandarizarse los extractos. Para la valoración se aplican técnicas volumétricas, gravimétricas, potenciométricas, espectrofotométricas - cromatográficas. De todas las anteriores, las cromatográficas son los más utilizadas, ya que hoy en día son las que generan resultados robustos, reproducibles y altamente confiables (Mohammad, Bhawani y Sharma, 2010). Ejemplo de ello es la cromatografía en capa delgada en su modalidad de alta resolución (HPTLC, por sus siglas en inglés), cromatografía de líquidos de alta eficiencia (HPLC, por sus siglas en inglés), cromatografía de líquidos de ultra eficiencia (UHPLC, por sus siglas en inglés) y cromatografía de gases (CG). En cuanto a la de líquidos de ultra eficiencia (UHPLC), pueden desarrollarse métodos que reduzcan significativamente el tiempo y el costo de análisis y de esta manera mejorar la calidad de los resultados (Pérez-Vásquez et al., 2014). Si lo que se necesita es estudiar un extracto que tiene compuestos volátiles (aceites esenciales, por ejemplo), la cromatografía de gases es la técnica de primera elección (Pérez-Vásquez et al., 2011). En muchos casos el uso de un equipo convencional de cromatografía de líquidos de alta resolución (HPLC) puede ser útil para los fines a los que se propone (Pérez-Vásquez et al., 2017).

En los últimos años se han implementado métodos cromatográficos más sofisticados como las técnicas acopladas, en virtud de que ofrecen información espectrométrica adicional, resultando de gran utilidad no sólo para el análisis cuantitativo, sino en la elucidación estructural. Entre estas técnicas destacan la cromatografía de líquidos acoplada a espectrometría de masas (UHPLC-MS, por sus siglas en inglés) (RiveroCruz et al., 2017), cromatografía de líquidos acoplada a resonancia magnética nuclear (HPLC-NMR) y cromatografía de gases acoplada a espectrometría de masas (CG-EM) (Sendker y Sheridan, 2017).

Finalmente, las determinaciones de pureza están orientadas a revelar la presencia de materia extraña, como tierra, arena o polvo. También en este ensayo se detecta la existencia de fragmentos de otras plantas ajenas o de la misma planta, pero que no corresponde a la droga cruda, así como de organismos o partes de ellos (por ejemplo, el gorgojo [Acanthoscelides obtectus] presente en el frijol). También se debe determinar la presencia de plaguicidas, en especial en aquellas plantas que provengan de cultivos cercanos a zonas de agricultura industrial, determinación de aflatoxinas, que son micotoxinas producidas por hongos principalmente del género Aspergillus y que generalmente están relacionados con condiciones de almacenamiento de alta humedad y alta temperatura (Peraica, Radić, Lucić y Pavlović, 1999), presencia de metales pesados (plomo, mercurio y arsénico, principalmente), así como de microorganismos en general.

\section{Extractos estandarizados y extractos cuantificados}

De las drogas crudas vegetales podemos obtener los extractos a partir de diferentes procesos como son maceración, percolación, infusión y decocción, por mencionar los más comunes (Azwanida, 2015). Estos extractos contendrán los marcadores y/o principios activos necesarios para ser utilizados en los ensayos de identidad química y en la valoración. Así, para asegurar a los consumidores de productos herbolarios que lo que están consumiendo contiene la planta indicada (identidad botánica), con los metabolitos que corresponden a su metabolismo (identidad química) y en la proporción correcta (ensayos de valoración), los 
extractos deben ser estandarizados de acuerdo con el contenido de los marcadores (Heinrich, Barnes, Gibbons y Williamson, 2012). Decimos, por lo tanto, que un extracto está estandarizado cuando contiene una proporción definida de uno o varios compuestos o grupos de compuestos; este valor se puede especificar en un rango, dado en porcentaje, que va de una cantidad mínima a una máxima, o en relación con un valor límite (un solo valor máximo permitido). Por lo tanto, la estandarización es requisito indispensable para asegurar la calidad respecto del contenido de metabolitos específicos, que serán los marcadores activos, así como la cantidad de droga cruda que se emplea para preparar los extractos (Yau, Goh y Koh, 2015).

La estandarización, en términos de producción, empieza desde el cultivo ya que es bien conocido que la variabilidad en la concentración de los metabolitos secundarios, así como su biosíntesis, inicia desde el crecimiento y desarrollo de la planta, donde factores agroecológicos (como la localización geográfica, el tiempo de cosecha, las estaciones del año, entre otros) influyen directamente en el contenido metabólico (Kumari y Kotecha, 2016). La estandarización también está relacionada con la forma del secado (si será pasiva o en hornos) y a los parámetros relacionados con la extracción como son el disolvente usado para obtener los extractos, el tiempo que tarde la extracción, la temperatura y el método (Pandey y Tripathi, 2014). La estandarización es pues, necesaria para garantizar la calidad de la planta medicinal como materia prima para productos herbolarios.

Por otra parte, en algunos casos no se tiene la certeza de cuál es el principio activo o se concluye, después de numerosos ensayos farmacológicos, que varios de ellos contribuyen a la actividad biológica; entonces se habla de extractos cuantificados. Por lo tanto, éstos hacen referencia a la determinación de cualquier tipo de marcador, sean activos, analíticos o negativos (Heinrich, Barnes, Gibbons y Williamson, 2012).

\section{Legislación sanitaria en} torno a los productos herbolarios

Se estima que hasta la fecha se encuentran documentadas 28,187 especies vegetales con usos medicinales y de éstas, sólo se encuentran inscritas en farmacopeas un porcentaje menor (aproximadamente $16 \%)$, lo que evidencia que se deben hacer mayores esfuerzos en la regulación para controlar y garantizar una mayor calidad de las plantas que serán usadas como productos herbolarios (Willis, 2017). Su inclusión en las farmacopeas permite regularlas para asegurar su calidad siguiendo estrictamente la metodología descrita en estos documentos oficiales. Al ser las plantas medicinales la materia prima para obtener un producto terminado de tipo herbolario, como los medicamentos y remedios, y en algunos casos suplementos alimenticios destinados a un uso terapéutico, nutricional y/o para el cuidado de la salud, es importante considerar la legislación sanitaria que los rige.

Los productos herbolarios que son considerados propiamente insumos para la salud son los medicamentos y los remedios herbolarios. Los primeros, al ser medicamentos como tal, están definidos en la Ley General de Salud (Secretaría de Salud, 2018) de la siguiente forma:

\begin{abstract}
Aquellos productos elaborados con material vegetal o algún derivado de éste, cuyo ingrediente principal es la parte aérea o subterránea de una planta o extractos y tinturas, así como jugos, resinas, aceites grasos y esenciales, presentados en forma farmacéutica, cuya eficacia terapéutica y seguridad ha sido confirmada científicamente en la literatura nacional o internacional (Secretaría de Salud, 2018, art. 224, Secc. B).
\end{abstract}

Los remedios herbolarios (RH) están citados y definidos en el Reglamento de Insumos para la Salud (RIS) como: 
[...] el preparado de plantas medicinales, o sus partes, individuales o combinadas y sus derivados, presentado en forma farmacéutica, al cual se le atribuye por conocimiento popular o tradicional el alivio para algunos síntomas participantes o aislados de una enfermedad (RIS, 1998, p. 22).

Tanto los remedios como los medicamentos herbolarios, y de acuerdo con la NOM-072-SSA1-2012, que rige el etiquetado de estos productos, requieren que la cantidad del principio activo y la equivalencia al marcador químico sea declarada en el marbete, lo que implica que se tengan que presentar como extractos estandarizados en relación con los principios activos (marcadores activos) seleccionados de acuerdo con la eficacia y seguridad de la especie vegetal.

Otro tipo de compuesto herbolario que puede ser considerado como tal, sólo si contiene entre sus ingredientes partes de una planta o extractos obtenidos de plantas, son los suplementos alimenticios, a los cuales la LGS define en el artículo 215 del capítulo segundo, dedicado a Alimentos y Bebidas no Alcohólicas:

Productos a base de hierbas, extractos vegetales, alimentos tradicionales, deshidratados 0 concentrados de frutas, adicionados o no, de vitaminas o minerales, que se puedan presentar en forma farmacéutica y cuya finalidad de uso sea incrementar la ingesta dietética total, complementarla o suplir alguno de sus componentes (Secretaría de Salud, 2018, p. 90).

Por lo que respecta a los tés, infusiones y tisanas, ni la LGS ni el RIS contemplan datos relacionados con su regulación; sin embargo, el Reglamento de Control Sanitario de Productos y Servicios (1999, p. 31), en su capítulo II, art. 130, sobre tés e infusiones, establece:

Tanto en la etiqueta como en la información con que se comercialicen los productos a que se refiere este capítulo no se deberá presentar información que confunda, exagere o engañe en cuanto a sus efectos, composición, origen y otras propiedades del producto, ni ostentar indicaciones rehabilitatorias o terapéuticas.

Adicional al reglamento citado, estos productos herbolarios deberán regirse bajo la Norma Oficial Mexicana NOM-051-SCFI/SSA1-2010, que fija las especificaciones generales de etiquetado para alimentos y bebidas no alcohólicas preenvasados. Por cuanto hace a la presentación de medicamentos y remedios herbolarios, la normatividad sanitaria permite que se utilicen las formas farmacéuticas incluidas en la Farmacopea de los Estados Unidos Mexicanos, y en el caso exclusivo de remedios herbolarios, además, se pueden presentar en forma de tinturas, extractos y tisanas (Secretaría de Salud 2013). Para las buenas prácticas de fabricación que inciden en la preparación y presentación comercial tanto de medicamentos como de remedios herbolarios, es de aplicación obligatoria la NOM-248-SSA1-2011: “Buenas prácticas de fabricación para establecimientos dedicados a la fabricación de remedios herbolarios".

Por otra parte, dentro del marco regulatorio para ser considerado en el comercio de plantas medicinales que serán usadas en preparados herbolarios, debe evitarse el empleo de aquellas que se encuentran enlistadas en el Apéndice II de la FHEUM ("Seguridad en el uso de plantas medicinales", Secretaría de Salud 2013) y también las que se incluyen en el "Acuerdo por el que se determinan las plantas prohibidas o permitidas para tés, infusiones y aceites vegetales comestibles" (Secretaría de Salud, 1999).

Además, se debe considerar el grado de riesgo ambiental de las plantas medicinales que serán utilizadas para comercializarse; es decir, conocer su estatus en torno a si están en peligro de extinción. En la NOM-059-SEMARNAT-2010, sobre Protección ambiental-Especies nativas de México de flora y fauna silvestres, viene una lista de plantas con su categoría de riesgo. 


\section{Conclusiones}

El control de calidad de plantas medicinales es un proceso de varios pasos que cubre todas las etapas de producción, desde la planta como materia prima hasta su empaque como producto terminado, ya sea medicamento o remedio herbolario. Desde el punto de vista de su aplicación, la calidad está encaminada primeramente a autentificar la especie vegetal; también conocer el contenido metabólico de las plantas es importante y fundamental, porque permite establecer cuál será el marcador que se empleará para los ensayos farmacopeicos. Es rigurosamente necesario que las plantas medicinales que se destinen a preparar productos herbolarios cumplan con las determinaciones analíticas específicas relacionadas con su calidad. En el caso de México, estas determinaciones se encuentran compiladas en la Farmacopea Herbolaria de los Estados Unidos Mexicanos (FHEUM), que es el documento oficial instituido para tal fin. Cualquier planta medicinal que deba utilizarse con fines terapéuticos para comercializarse, o que incida en aspectos de la salud de la población en general, tendrá que demostrar su calidad de acuerdo con los procedimientos oficiales enmarcados en la FHEUM (parámetros de identidad, composición y pureza), así como en toda la normatividad vigente relacionada con la regulación de productos herbolarios. Finalmente, es importante mencionar que aún falta por incorporar muchas plantas medicinales de amplio uso entre la población mexicana en la FHEUM y por lo tanto todos los involucrados en el estudio, manejo, preservación o difusión de plantas medicinales debemos tomar un rol más activo. Esto se puede lograr contribuyendo en la generación de información científica que respalde la calidad y que refleje la realidad nacional en torno a la extensa flora medicinal de nuestro país.

\section{Referencias}

Ahmed, S. y Hasan, M. M. (2015). Crude drug adulteration: a concise review. World Journal of Pharmacy and Pharmaceutical Sciences, 4(10), 274-283.
Antonio Barrera, N. (1992). Plantas medicinales usadas en la comunidad de San Juan Guichicovi. En P. Sesia (ed.), Medicina Tradicional, Herbolaria y Salud Comunitaria en Oaxaca. México: Gobierno del Estado de Oaxaca/ CIESAS Pacífico.

Azwanida, N. N. (2015). A Review on the extraction methods use in Medicinal Plants, principle, strength and limitation. Medicinal \& Aromatic plants, 4, 196-201.

Cadena-Iñiguez, P., Cruz-Morales F., D. C. y BallinasAlbores, E. (2014). Tepezcohuite (Mimosa tenuiflora (L) Willd) el árbol de la piel. AP Agro Productividad, 7(6), 10-16.

Calahan, J., Howard, D., Almalki, A. J., Gupta. M. P. y Calderón A., I. (2016). Chemical adulterants in herbal medicinal products: a review. Planta Médica, 82(6), 505-515.

Clevenger, J. F. y Ewing, C. O. (1919). Santolina chamaecyparissus L, an adulterant of Matricaria chamomilla L. The Journal of the American Pharmaceutical Association, 8(7), 536-538.

Croteau, R., Kutchan, T. M. y Lewis, N. G. (2000). Natural Products (Secondary Metabolites). En B. B. Buchanan, W. Gruissem y R. L. Jones (eds.), Biochemistry \& molecular biology of plants (pp. 1250-1318). Rockville, Md.: American Society of Plant Physiologists.

Das, M. (2014). Chamomile: medicinal, biochemical, and agricultural aspects. Boca Raton: CRC Press.

Delgado, G., Olivares, M. S., Chávez, M. I., RamírezApan T., Linares E., Bye, R. y Espinosa-García, F. J. (2001). Antiinflammatory Constituents from Heterotheca inuloides. Journal of Natural Products, 64(7), 861-864.

Evans, C. E. (2009). The scope and practice of pharmacognosy. En Trease and Evans pharmacognosy (pp. 5-7). Edinburgh: Saunders/Elsevier.

Foster, S. (2011). A brief history of adulteration of herbs, spices, and botanical drugs. Herbal Gram, 92, 42-57.

Gafner, S. y Applequist, W. (2016). On Adulteration of Arnica montana. Botanical Adulterants Bulletin, 1-5. 
Gómez Castellanos, J. R. (2009). El ambiente regulatorio de los medicamentos herbolarios en México. Antecedentes, situación actual y perspectivas al año 2025. Boletín Latinoamericano y del Caribe de Plantas Medicinales y Aromáticas, 8(1), 33-40.

Guzelmeric, E., Vovk, I. y Yesilad, E. (2015). Development and validation of an HPTLC method for apigenin 7-0-glucoside in chamomile flowers and its application for fingerprint discrimination of chamomile-like materials. Journal of Pharmaceutical and Biomedical Analysis, 107, 108-118.

Heinrich, M., Barnes, J., Gibbons, S. y Williamson, E. M., (eds.). (2012). Production, standardization and quality control. En Fundamentals of Pharmacognosy and Phytotherapy (pp. 144-161). Edinburgh: Churchill Livigstone, Elsevier.

Khan, I. A. y Smillie, T. (2012). Implementing a "Quality by Design" Approach to Assure the Safety and Integrity of Botanical Dietary Supplements. Journal of Natural Products, 75(9), 1665-1673.

Kumari, R. y Kotecha, M. (2016). A review on the Standardization of herbal Medicines. International Journal of Pharma Sciences and Research, 7(2), 97-106.

Li, S., Han, Q., Qiao, C., Song, J., Cheng, L. C. y Xu, H. (2008). Chemical markers for the quality control of herbal medicines: an overview. Chinese Medicine, 3(7). doi: 10.1186/1749-8546-3-7

Mariotti, K., C., Rossato, L. G., Fröehlich, P. E. y Limberger, R. P. (2013). Amphetamine-Type Medicines: A Review of Pharmacokinetics, Pharmacodynamics, and Toxicological Aspects. Current Clinical Pharmacology, 8(4), 350-357.

Martínez P., F. D. y López Herrera, A. (1995). Propagación sexual del tepezcohuite (Mimosa tenuiflora (Wild). Horticultura, 4, 135-138.

Mitra, S. K., y Kannan, R. (2007). A note on unintentional adulterations in Ayurvedic herbs. Ethnobotanical Leaflets, 11, 11-15.

Mohammad, A., Bhawani, S. A. y Sharma, S. (2010). Analysis of herbal products by thin-layer chromatography: a review. International Journal of Pharma and Bio Sciences, 1(2), PS16.
Norma Oficial Mexicana NOM-001-SSA1-2010, Que instituye el procedimiento por el cual se revisará, actualizará y editará la farmacopea de los Estados Unidos Mexicanos. Diario oficial de la Federación 26/01/2011.

Norma Oficial Mexicana NOM-051-SCFI/SSA1-2010, Especificaciones generales de etiquetado para alimentos y bebidas no alcohólicas preenvasadosInformación comercial y sanitaria. Diario oficial de la Federación 05/04/2010.

Norma Oficial Mexicana NOM-059-SEMARNAT-2010, Protección ambiental-Especies nativas de México de flora y fauna silvestres-Categorías de riesgo y especificaciones para su inclusión, exclusión o cambio-Lista de especies en riesgo. Diario Oficial de la Federación, 6/11/10.

Norma Oficial Mexicana NOM-072-SSA1-2012, Etiquetado de medicamentos y de remedios herbolarios, Diario Oficial de la Federación 21/11/2012.

Norma Oficial Mexicana NOM-248-SSA1-2011, Buenas prácticas de fabricación para establecimientos dedicados a la fabricación de remedios herbolarios.

Organización Mundial de la Salud (OMS). (2002). Estrategia de la OMS sobre medicina tradicional. 2002-2005. Ginebra: autor. Disponible en: http:// www.sld.cu/galerias/pdf/sitios/mednat/estrategia_ de_la_oms_sobre_medicina_tradicional.pdf

OMS. (2011). Quality control methods for herbal materials. Updated edition of Quality control methods for medicinal plant materials. Malta: autor.

OMS. (2013). Estrategia de la OMS sobre medicina tradicional, 2014-2023. Ginebra: autor. Disponible en http://web.minsal.cl/sites/default/files/files/ s21201es.pdf

Ortega, P., Phail, E. y Vega Montiel, A. (2011). Productos milagro y medios de comunicación en México: una reflexión crítica. Derecho a Comunicar, 3, 104-117.

Owoyele, V. B., Wuraola, C. O., Soladoye, A. 0. y Olaleye, S. B. (2004). Studies on the antiinflammatory and analgesic properties of Tithonia 
diversifolia leaf extract. Journal of Ethnopharmacoly, 90(2-3), 317-321.

Pandey, A. y Tripathi, S. (2014). Concept of standardization, extraction and pre phytochemical screening strategies for herbal drug. Journal of Pharmacognosy and Phytochemistry, 2(5), 115-119.

Parvathy, V. A., Swetha, V. P., Sheeja, T. E. y Sasikumar, B. (2015). Detection of plant-based adulterants in turmeric powder using DNA barcoding. Pharmaceutical Biology, 53(12), 1774-1779.

Peraica, M., Radić, B., Lucić, A. y Pavlović, M. (1999). Toxic effects of mycotoxins in humans. Bulletin of the World Health Organization, 77(9), 754-766.

Pérez-Vásquez, A., Capella, S., Linares, E., Bye, R., Angeles-López, G. y Mata, R. (2011). Antimicrobial activity and Chemical composition of the essential oil of Hofmeisteria schaffneri. Journal of Pharmacy and Pharmacology, 63, 579-586.

Pérez-Vásquez, A., Castillejos-Ramírez, E., Cristians, S. y Mata, R. (2014). Development of an UPLC-PDA method for the simultaneous quantification of 4-phenylcoumarins and chlorogenic acid in Exostema caribaeum stem bark. Journal of Natural Products, 77, 516-520.

Pérez-Vásquez, A., Ángeles-López, G., Rivero-Cruz, I., Flores-Bocanegra, L., Linares, E., Bye, R. y Mata, R. (2017). Spasmolytic Action of Preparations and Compounds from Hofmeisteria schaffneri. Natural product Communications, 12(4), 475-476.

Posadzki, P., Watson, L. y Ernst, E. (2013). Contamination and adulteration of herbal medicinal products (HMPs): an overview of systematic reviews. European Journal of Clinical Pharmacology, 69(3), 295-307.

Rajani, M. y Kanaki, N. S. (2008). Phytochemical Standardization of Herbal Drugs and Polyherbal Formulations. En K.G. Ramawat y J. M. Mérillon (eds.), Bioactive Molecules and Medicinal Plants (pp. 349369). Berlín: Springer Verlag.

Reglamento de Control Sanitario de Productos y Servicios. (1999). (Última reforma publicada en 2016). México: Secretaría de Salud. Disponible en http://www.ordenjuridico.gob.mx/Documentos/ Federal/pdf/wo88301.pdf
Rivera-Arce, E., Gattuso, M., Alvarado, R., Zárate E. J., Agüero, J., Feria, I. y Lozoya, X. (2007). Pharmacognostical studies of the plant drug Mimosae tenuiflorae cortex. Journal of Ethnopharmacology, 113(3), 400-408.

Rivero-Cruz, I., Anaya-Eugenio, G., Pérez-Vásquez, A., Martínez, A. L. y Mata, R. (2017). Quantitative analysis and pharmacological effects of Artemisia ludoviciana aqueous extract and compounds. Natura I Product Communications, 12, 1531-1534.

Rocha, T., Amaral, J. S. y Oliveira, M. B. P. P. (2016). Adulteration of Dietary Supplements by the Illegal Addition of Synthetic Drugs: A Review. Comprehensive Reviews in Food Science and Food Safe, 15(1), 43-62.

Rosas-Acevedo, H., Terrazas, T., González-Trujano, M. E., Guzmán, Y. y Soto Hernández, M. (2011). Antiulcer activity of Cyrtocarpa procera analogous to that of Amphipterygium adstringens, both assayed on the experimental gastric injury in rats. Journal of Ethnopharmacology, 134(1), 67-73.

Salim, A. A., Chin, Y. W. y Kinghorn, A. D. (2008). Drug Discovery from Plants. En K.G. Ramawat y J. M. Mérillon (eds.), Bioactive Molecules and Medicinal Plants (pp. 1-24). Berlín: Springer Verlag.

Secretaría de Salud. (1999). Acuerdo por el que se determinan las plantas prohibidas o permitidas para tés, infusiones y aceites vegetales comestibles. México: Diario Oficial de la Federación. Disponible en: http://www.salud.gob.mx/unidades/cdi/nom/compi/ a1512993.html

Secretaría de Salud. (2013). Farmacopea Herbolaria de los Estados Unidos Mexicanos (FHEUM). México: autor.

Secretaría de Salud. (2018). Ley General de Salud (LGS), última reforma publicada. México: Diario Oficial de la Federación. Disponible en http://www.ordenjuridico. gob.mx/Documentos/Federal/pdf/wo11037.pdf

Selvam, A. B. D. (2010). Is the term substitution relevant to Pharmacognosy and/ or vegetable crude drug industry? Pharmacognosy Research, 2(5), 323-324.

Sendker, J. y Sheridan, H. (2017). Composition and Quality Control of Herbal Medicines. En O. Pelkonen, P. Duez, P. M. Vuorela y H. Vuorela (eds.), Toxicology of Herbal Products (pp. 29-65). Cham, Suiza: Springer. 
Srirama, R, Santhosh, Kumar, J. U, Seethapathy, G. S, Newmaster, S. G., Ragupathy, S., Ganeshaiah, K. N., Uma Shaanker, R. y Ravikanth, G. (2017). Species Adulteration in the Herbal Trade: Causes, Consequences and Mitigation. Drug Safety, 40(8), 651-661.

Yau, W-P., Goh, C. H. y Koh, H-L. (2015). Quality Control and Quality Assurance of Phytomedicines: Key Considerations, Methods, and Analytical Challenges. En I. Ramzan (ed.), Phytotherapies: Efficacy, Safety, and Regulation (pp. 18-48). Hoboken: John Wiley \& Sons Inc.

Waizel-Bucay, J. y Cruz-Juárez, M. L. (2014). Arnica montana L., planta medicinal europea con relevancia. Revista Mexicana de Ciencias Forestales, 5(25), 98-109.
Williams, D. H., Stone, M. J., Hauck, P. R. y Rahman, S. K. (1989). Why Are Secondary Metabolites (Natural Products) Biosynthesized? Journal of Natural Products, 52(6), 1189-1208.

Willis, K. J. (Ed.). (2017). Useful plants-medicines. En State of the World's Plants (pp. 22-29). Londres: Royal Botanic Gardens.

Wosch, L., Imig, D. C., Cervi, A. C., Moura, B. B., Budel, J. M. y de Moraes Santos, C. A. (2015). Comparative study of Passiflora taxaleaves: I. A morpho-anatomic profile. Revista Brasileira de Farmacognosia, 25(4), 328-343.

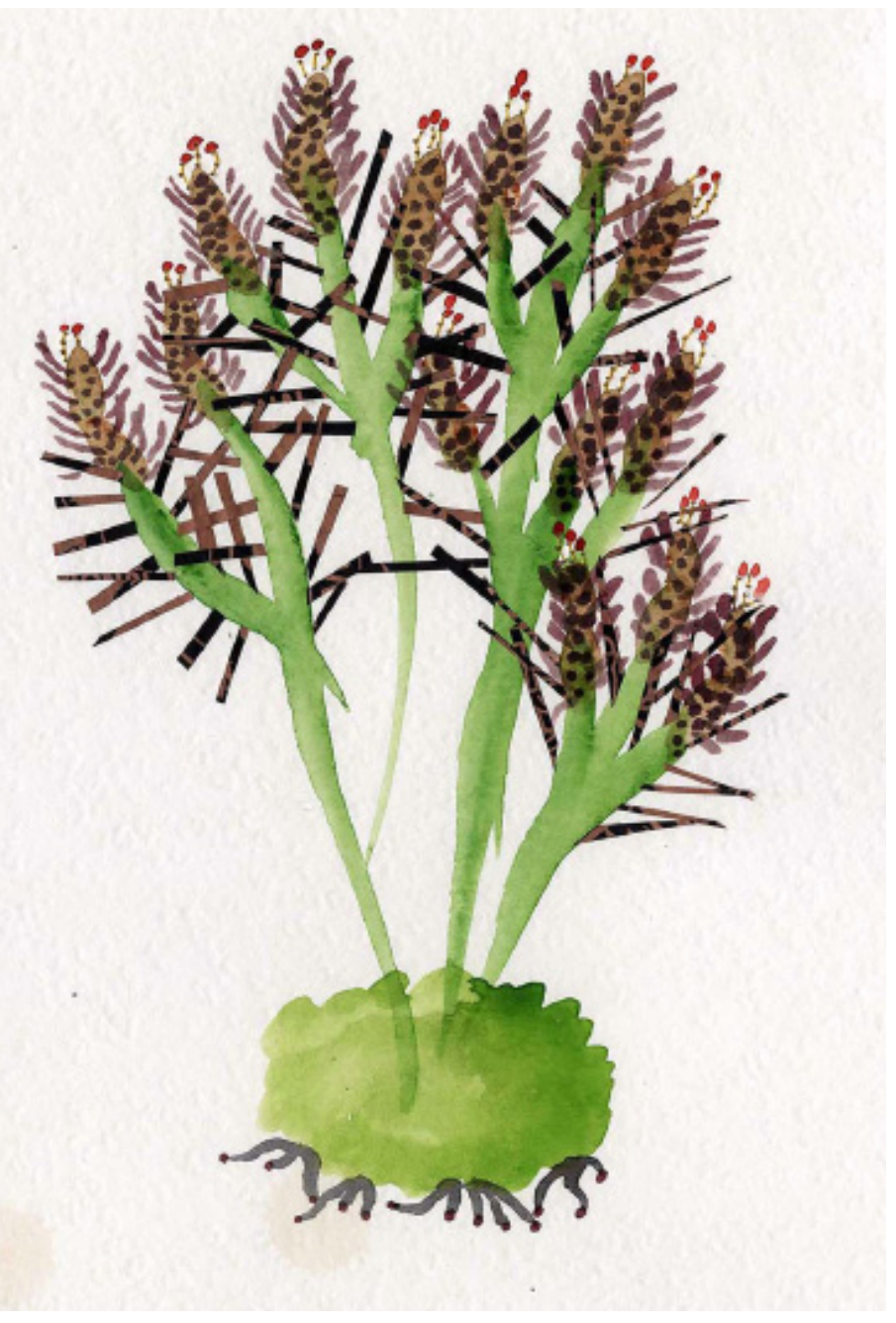

"Quappoquietl canyon"

Acuarela, fragmentos de bolsas de papel de productos de consumo y bordado sobre papel de algodón. $26 \times 18 \mathrm{~cm}$ 2017 